XI.

Aus der II. chirurg. Abteilung: Vorstand Hofrat v. Mos etigMoorhof, und dem Röntgenlaboratorium: Vorstand Dozent G. Holzknecht des k. k. allgem. Krankenhauses in Wien.

\title{
Eine bisher nicht beobachtete kongenitale, hereditäre Anomalie des Fingerskelettes.
}

\author{
Von \\ cand. med. Bernhard Bauer.
}

(Mit 6 Abbildungen.)

An der II. chirurgischen Abteilung kam folgender Fall von kongenitaler Fingeranomalie zur Beobachtung, der mir für die Kasuistik der Abnormitäten aus zwei Gründen bemerkenswert erscheint: Zunächst, weil ein derartiger Fall bisher noch nicht beobachtet wurde, und dann besonders deshalb, weil sich die zu beschreibende Anomalie nicht auf ein einzelnes Individuum beschränkt, sondern auf hereditärer Grundlage basiert und ungemein zahlreiche Personen der Familie betrifft.

Es handelt sich um einen aus sonst gesunder Familie stammenden Patienten, Ferdinand K., 15 Jahre alt, Kürschnerlehrling, der an obgenannter Abteilung wegen mehrfacher Panaritien in Behandlung stand. Die Anomalie, welche mir bei der ambulatorischen Behandlung des Kranken auffiel, beschränkt sich auf den kleinen Finger beider Hände; die Untersuchung ergab folgenden klinischen Befund:

Rechts: Der kleine Finger erscheint zunächst relativ verkürzt, indem die Spitze desselben nicht dem zweiten Interphalangealgelenk des vierten Fingers entspricht, sondern ungefähr der Mitte der zweiten Phalanx (siehe Fig. 1). Auch beschreibt der kleine Finger einen ulnarwärts konvexen Bogen, so zwar, daß die Radialseite des kleinen Fingers und die Ulnarseite des vierten Fingers einen ca. $3 \frac{1}{2} \mathrm{~cm}$ langen Schlitz miteinander einschließen. Bei genauerer Analyse des klinischen Bildes ergibt sich Folgendes: Die Grundphalanx des Fingers bietet nichts Abnormes; die Mittelphalanx hingegen erscheint bedeutend verkurzt und von keilförmiger Ge- 
Eine bish. nicht beobachtete kongen., hereditäre Anomalie d. Fingerskel. 253

stalt mit ulnarer Basis, was sich schon aus der Konvergenz der den beiden Interphalangealgelenken entsprechenden dorsalen, Hautfurchen erkennen läBt. Die Endphalanx zeigt in ihrem Bau nichts Abnormes, weicht nur infolge der schrägen, proximalen Fläche der Mittelphalanx stark radialwärts ab. Sonst wäre noch bemerkenswert, dab die Endphalanx sich im zweiten Interphalangealgelenk ganz bedeutend überstrecken läBt. - Links derselbe Befand in geringerem MaBe.

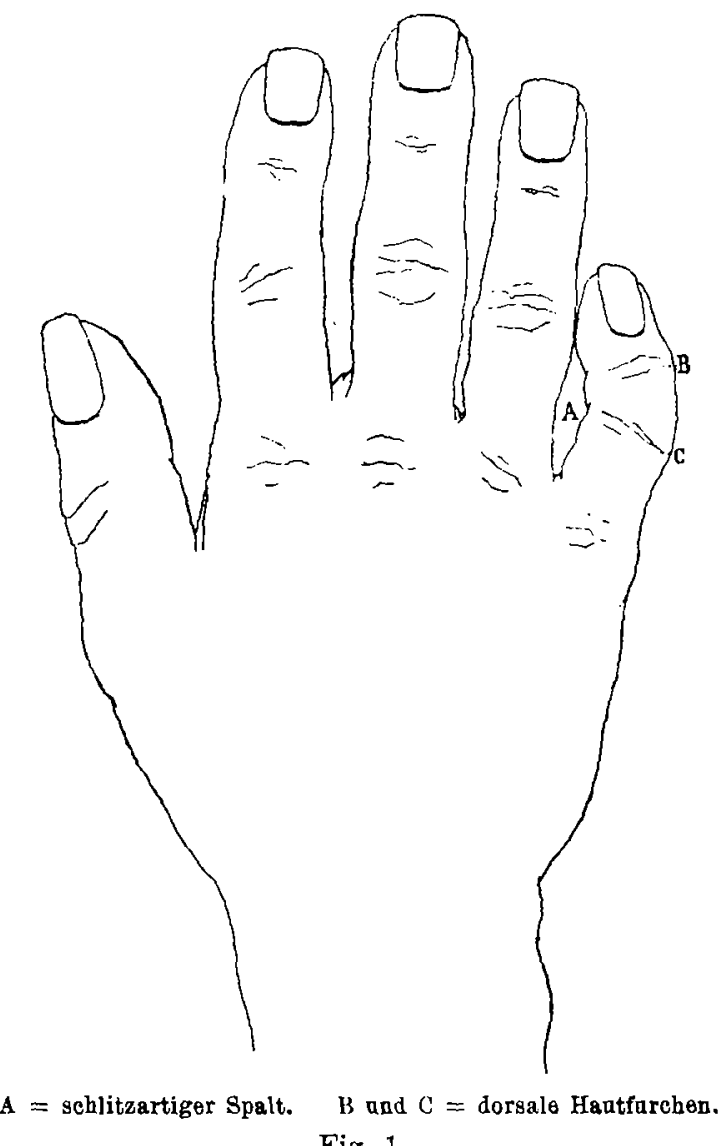

Fig. 1.

Die Röntgenuntersuchung, welche ich zur Aufklärung des klinischen Befundes veranlaBte, lieB uns die im Folgenden zu beschreibenden Anomalien des Fingerskelettes erkennen.

Die Grundphalanx des kleinen Fingers zeigt bei genauester Práfung keine, wie immer geartete Abweichung von der Norm, sowohl in Bezug auf GröBe als auch Struktur. Die Endphalanx hingegen zeigt im Vergleiche mit anderen, gleichaltrigen Individuen angehörigen eine relative Verlängerung, welche ungefähr $1 / 2 \mathrm{~cm}$ beträgt und im Skelette der rechten 
Hand deutlicher ausgeprägt erscheint als in dem der linken. Auch die normalerweise der Endphalanx eigene, hufförmige Endauftreibung ist nicht so deutlich ausgeprägt wie an den andern Fingern (Figur 2 und 3).

Die Mittelphalanx, der eigentliche Sitz der Anomalie, macht zunächst den Eindruck eines aus zwei bis drei Anteilen zusammengesetzten, im

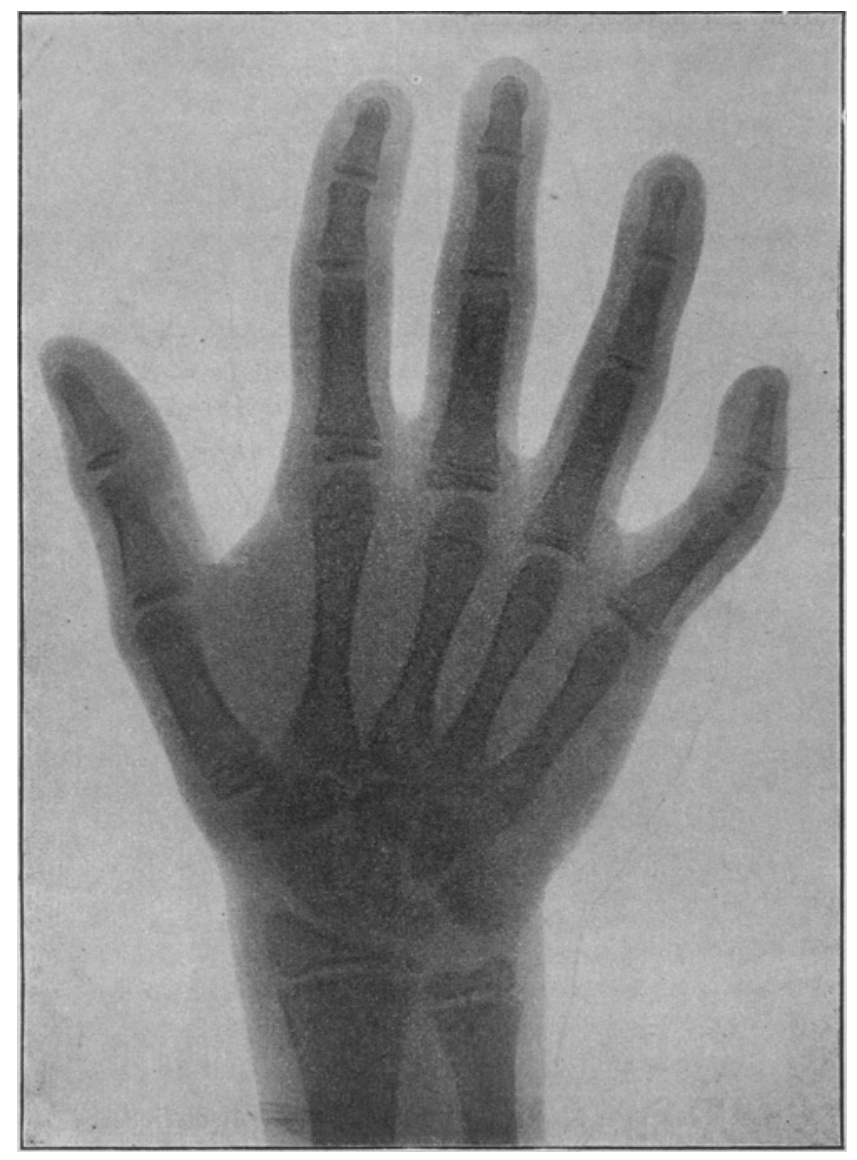

Fig. 2.

Långsschnitte trapezähnlich geformten Körpers, dessen radiale uud ulnare Flächen parallel verlaufen, während die proximale und distale Endfläche ulnarwärts divergieren, und zwar so, daB der Winkel zwischen ulnarer und proximaler Kante nahezu ein rechter, zwischen ulnarer und distaler dagegen ein spitzer ist. Als Folge dieses Umstandes ergibt sich, daB die Achse zwischen End- und Grundphalanx keine geradlinige ist, sondern im ¿weiten Interphalangealgelenk geknickt erscheint, ein Befund, der die Kon- 
Eine bish. nicht beobachtete kongen., hereditäre Anomalie d. Fingerskel.

vergenz der fruher erwähnten, dorsalen Hautfalten entsprechend dem ersten und zweiten Interphalangealgelenk erklärt. Bei genauerer Analyse der die Mittelphalanx konstituierenden Skeletteile ergab es sich, daB man deutlich drei Knochenstucke erkennt, deren zwei - distal und proximal gelegen - die Form schalenartig geẅ̈lbter, nahezu parallelwandiger Knochen-

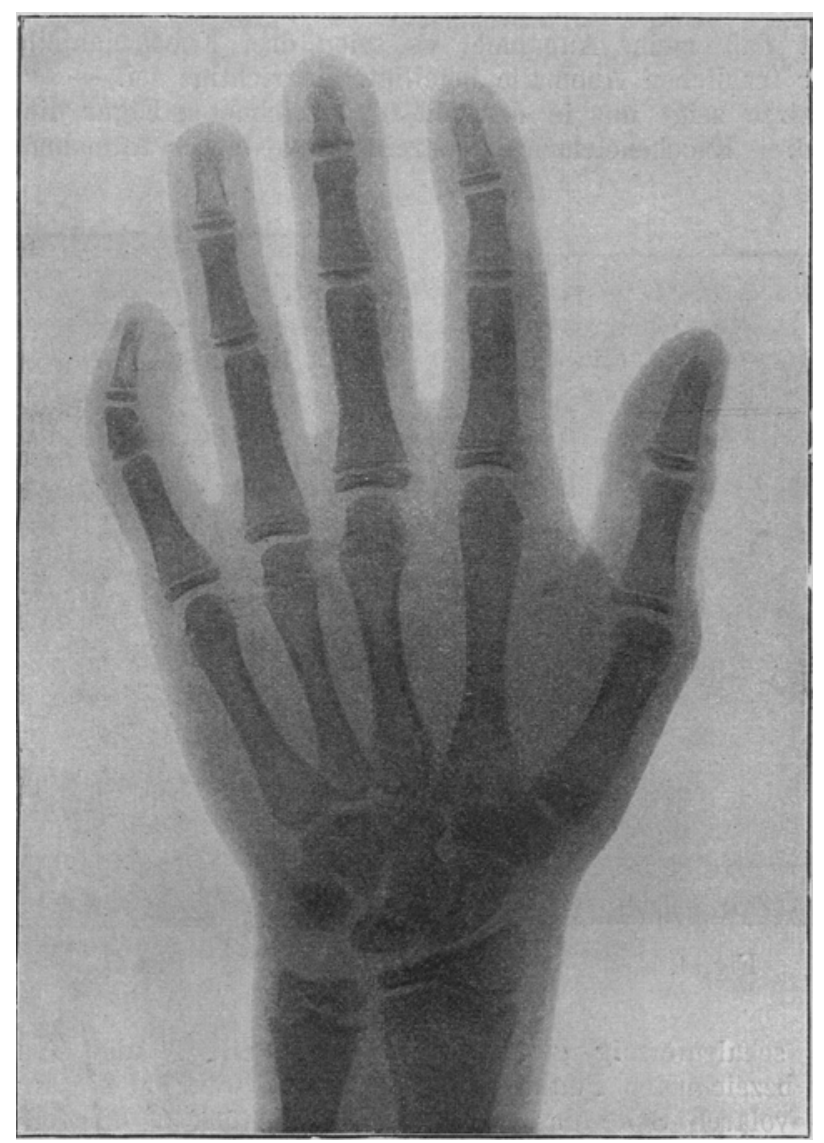

Fig. 3.

platten besitzen. Diese sind in einem ulnarwärts offenen Winkel gegeneinander gelagert und beherbergen in dem so gebildeten Spatium einen dritten Knochenanteil von unregelmäBig, dreieckiger Form mit radialer Spitze und ulnar gelegener Basis. Zwischen diesen drei Anteilen finden sich zwei Spatia in dem Sinne, als wäre der mittlere Anteil gleich einem Kerne exzentrisch in die beiden anderen Knochenstäcke eingekeilt. Im Skelette der rechten Hand weisen die beiden vorerwähnten, parallelwan- 
digen Knochenelemente - distal und proximal gelegen - radialwärts je einen spitzen, etwas gekrummten Fortsatz auf, die sich nahezu bertihren, während links ähnliche Fortsätze wohl angedeutct erscheinen, die gegenseitige Berthrung derselben jedoch noch nicht erfolgt ist. Bei Betrachtung der rein seitlichen Radiogramme (Figur 4 und 5) imponiert zwar die Mittelphalanx als aus nur zwei Knochenanteilen gebildet. Bei genauerer Analyse jedoch ergab sich im Verein mit nebenstehender rein geometrischer Überlegung, daB meine Annahme, es seien drei Knochenanteile an der Bildung der fraglichen Anomalie beteiligt, die richtige sei. -

Die Skizze zeigt uns in der mit $0^{0}$ bezeichneten Figur die Verhältnisse der drei Knochenelemente bei rein dorso-volarer Aufnahmsrichtung.

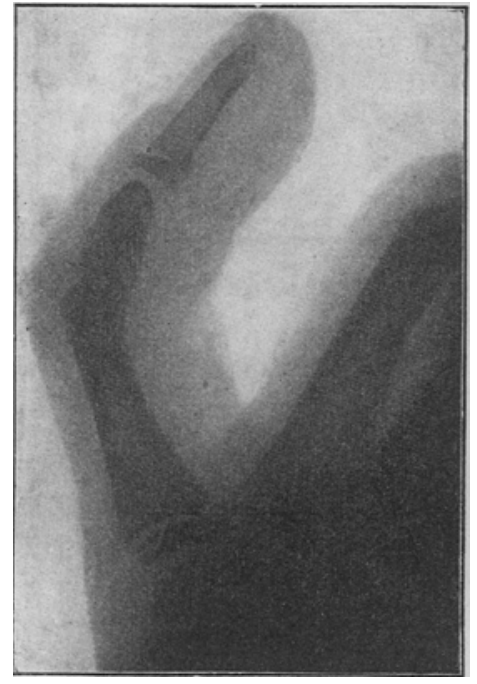

Fig. 4.

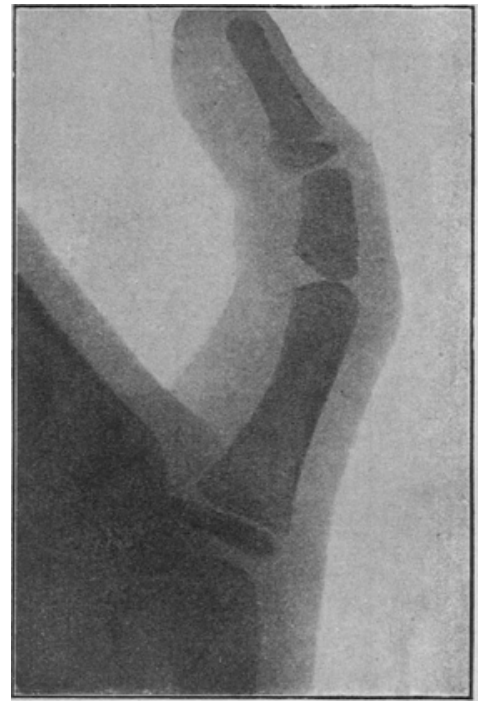

Fig. $\overline{5}$.

Die beiden schalenförmig gewölbten Knochenanteile $A$ und $B$ treffen in dem mit $a$ bezeichneten Punkte hart aneinander, während sie an der hinteren, also volaren Seite das mittlere Knochenstuck $C$ umgreifen. Auf rein geometrischer Konstruktion beruhend, wurde nun die zweite Skizze im Sinne einer Drehung um $90^{\circ}$ verfertigt und erwies sich als vollkommen korrespondierend mit den seitlich - radio-ulnarwärts gemachten - Röntgenaufnahmen. Der bei dorso-volarer Aufnahme dem Punkte $a$ entsprechende Schlitz verschwindet bei einer Drehung um $90^{\circ}$ naturgemäB, da sich ja nunmehr die beiden Knochenelemente $A$ und $B$ hart aneinder legen. Der dem distalen Teile angehörende, tiefste Punkt $d$ rückt nun an die Grenze der Knochenteile $A$ und $B$, ja er nimmt sogar an dem Mittelstacke $C$ Anteil. Von diesem selbst ist nur die distale Hälfte deutlich sichtbar, da ja proximalwärts das Knochenelement $B$ uber $C$ gelagert erscheinen muB. 
Bei rein volar-dorsaler Aufnahmsrichtung, die einer Drehung von $180^{\circ}$ in toto entsprechen würde, müBten die sich gegenseitig nicht deckenden Teile der drei Knochenstücke als schmale Streifen imponieren, während der weitaus größte Teil die genaueren Umrisse verschwinden ließe und mit diesen auch die Punkte $a$ und $d$.

Ehe ich an die Erklärung dieses so interessanten Falles gehe, muß ich in Kürze einige normal-anatomische Details bezüglich eines noch nicht vollkommen entwickelten Fingerskelettes anführen. Die Knochen der Finger gehen aus je einer Diaphyse und einer Epiphyse hervor. Die letztere bildet bei den Mittelhandknochen das Köpfchen, bei den Phalangen hingegen die Basis. Die Epiphysen-

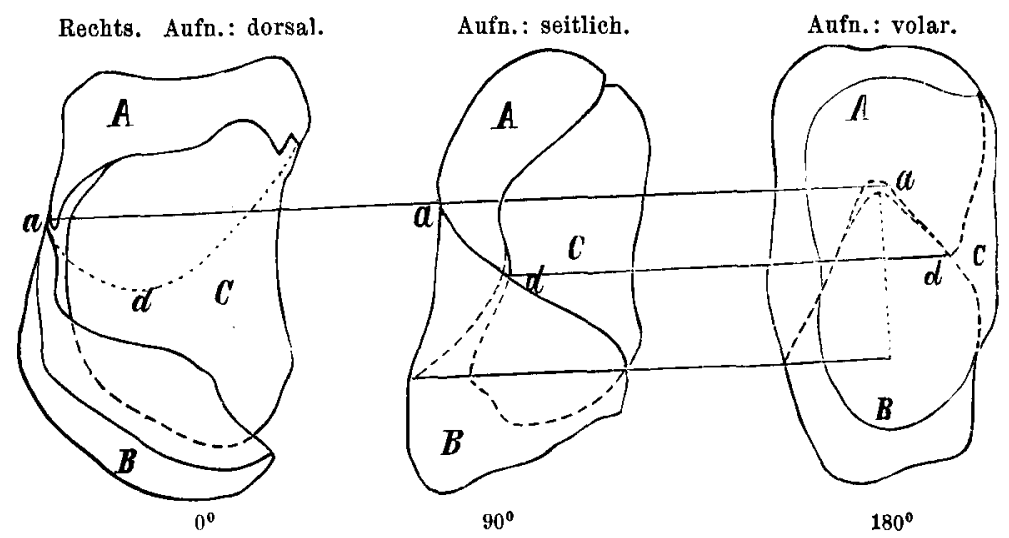

Fig. 6.

kerne erscheinen gegen Ende des 2. oder zu Anfang des 3. Lebensjahres und verschmelzen im 17. oder 18. Lebensjahre mit den entsprechenden Diaphysen. Die Mittelstücke der Fingerknochen beginnen in der 10. bis 14. Woche des Embryonallebens zu verknöchern, und zwar zuerst die der Phalanx prima und tertia, etwas später erst die der Phalanx media, wobei der Zeigefinger den Anfang, der kleine Finger den Schluß macht ( $\nabla$. Langer-Toldt).

Da es sich nun in unserem Falle um ein 15 jähriges Individuum handelt, sollten wir entsprechend den eben geschilderten Details, gleich den anderen Fingern auch an der Phalanx II des kleinen Fingers eine noch nicht verschmolzene Epiphyse sowie eine Diaphyse erwarten. Statt dieser beiden Knochenelemente, die ja isolierten Ossifikationspunkten entsprechen, finden wir nun drei Knochenkerne und zwei Zwischenknorpel, die in der oben beschriebenen Weise die Phalanx II bilden. Wir finden also eine Vermehrung der 
Ossifikationskerne. Da nun die Struktur der distalen und proximalen Knochenplatte völlig gleichmäßig ist und ihrer Dichte nach der aller übrigen Epiphysen vergleichbar erscheint, während der eingeschlossene Knochenkern seiner Struktur nach wabig zerklüftet, also von abnormalem Aufbau erscheint, glaube ich annehmen zu dürfen, daß es sich in dem vorliegenden Kasus um eine supernumeräre, distale Epiphyse handelt. Auch der schalenförmige, konkav gehöhlte Aufbau der beiden Knochenplatten leitet mich zu dieser Annahme. Finden wir doch bei den meisten Epiphysen des menschlichen Skelettes stets eine konkav gehöhlte Fläche vor. Das mittlere, dreikantige Knochenstïck wäre dieser Anschauung gemäß als Diaphyse aufzufassen, welche sowohl in ihrem Längenwachstum zurückgeblieben ist, als auch einen abnormalen inneren Aufbau angenommen hat. So müßte späterhin in völlig ausgebildetem Zustand die Phalanx II als keilförmiger Knochen imponieren, eine Vermutung, die sich schon jetzt durch den rein klinischen Befund bestätigt.

\section{Grobvater $80 \mathrm{~J}$.}

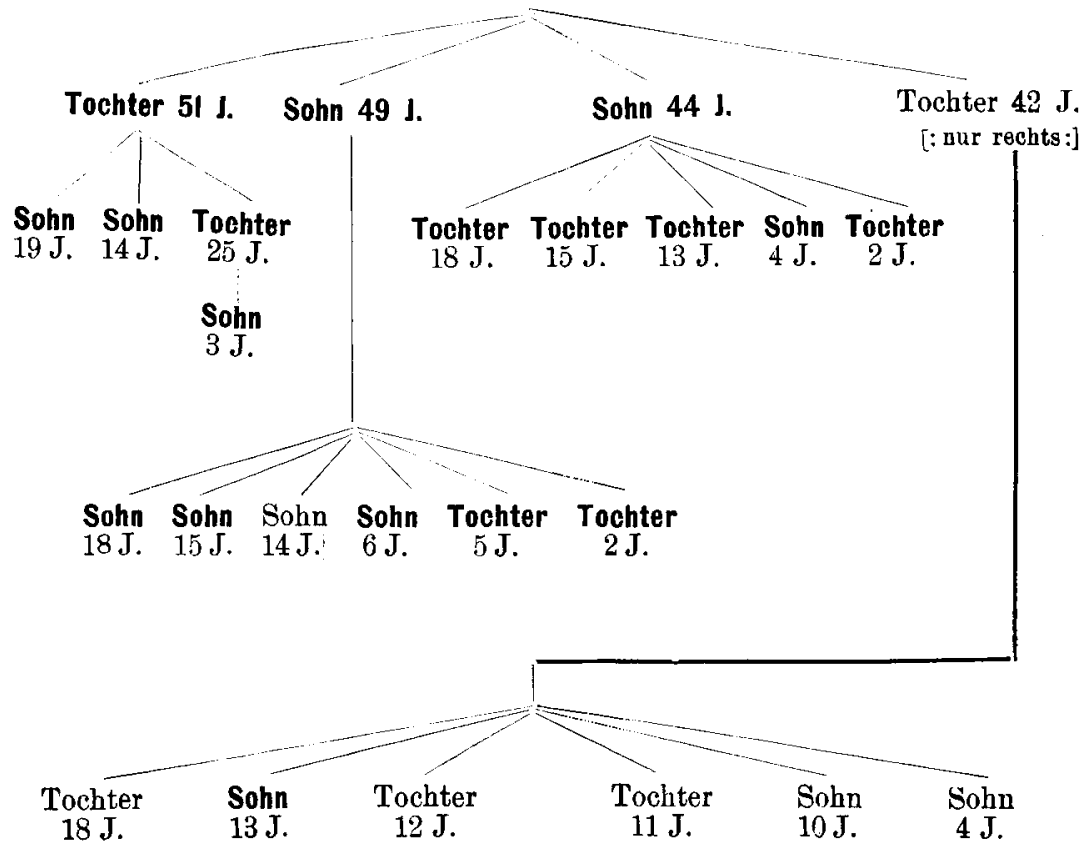

Leider war es mir nicht möglich als Beweis der Richtigkeit meiner Annahme mehrere Individuen der mit dieser Anomalie behafteten Familie untersuchen zu können. Die auf mündliche Mit- 
Eine bish. nicht beobachtete kongen., hereditäre Anomalie d. Fingerskel. 259

teilung des Patienten und schriftliche Auskunft des Vaters desselben erhaltene, vorstehende Familienstatistik beweist es jedoch zur Genüge, daß die abnormale Krümmung des kleinen Fingers auch in höherem Alter eine bleibende sei und hereditären Charakter trägt. ${ }^{1}$ )

Dr. G. Joachimsthal veröffentlichte in seinem Atlas kongenitaler Verbildungen der oberen Extremitäten das Röntgenogramm eines Falles von Brachydaktylie und Hyperphalangie, das auch eine eigenartige Krümmung des kleinen Fingers aufweist und so bei oberflächlicher Betrachtung Ähnlichkeit mit dem von mir beschriebenen Kasus aufweisen könnte. Nach Bericht dieses Autors ist jedoch das eigentümliche Verhalten des kleinen Fingers bloß auf eine Kontraktur zurückzuführen. -

1) Die mit der Anomalie behafteten Familienmitglieder - fett gedruckt. 